\title{
Analysis of the Digital Creation Spirit of Art works
}

\author{
Jing Li \\ Heilongjiang University of Technology, Jixi, Heilongjiang 158100, China
}

Keywords: digital technology; art works; artistic spirit

\begin{abstract}
With the upgrading of Internet bandwidth and the maturity of digital presentation technology, the creation and display of art works has begun to move from physical objects to digitalization. Digital art continues to create amazing works, leading to a huge and profound transformation in the field of visual arts creation. This paper preliminary summarizes theoretically the creation characteristics and artistic features of digital art, and then from a deeper level, makes a certain excavation of the artistic spirit and humanistic value of digital art, finally, describes some reflections brought about by digital art works.
\end{abstract}

\section{Introduction}

As people are more and more rich in material life, enriching the demands of spiritual life will become the main demand of people's life. With the rapid development of Internet technology and computer technology, the creation and display of works of art also started on the Internet, and the art works in the network also begin to present new manifestations and connotations.

Digital art works are the combination of modern technology and art. Computers can not only imitate some complicated traditional painting techniques, but also create a lot of new tools, so it makes digital art works show some different visual effects in traditional Chinese art works. Moreover, its convenient replicating and modifying functions make the art creation more simple and efficient. Compared with traditional art, the artistic charm of the digital art works is unique, and it is more in line with the aesthetic heart of modern people. Nowadays, the scope of the creation of digital art has covered every field of applied art and pure art, from plane to three dimensional, from static visual works to film and television animation, the great charm and strong vitality of digital art are everywhere. Digital technology has become an indispensable tool for creation in the field of visual arts, and attracts more and more art creators to join in.

\section{Artistic Spirit of Digital Art Works}

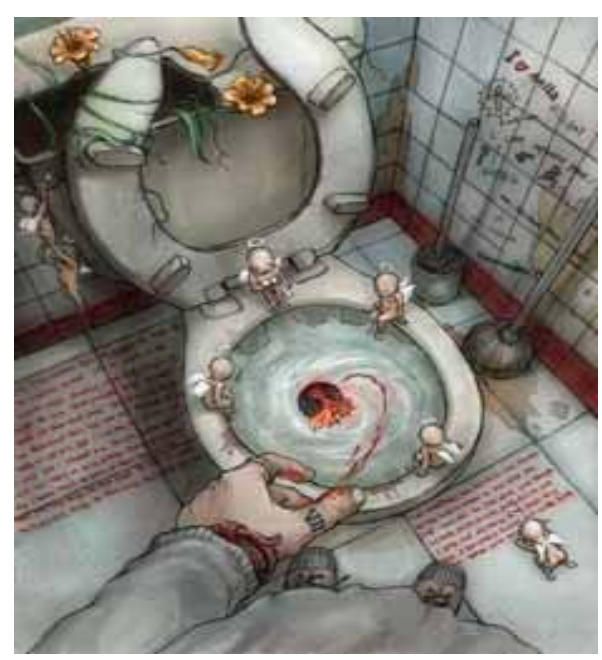

Figure 1. Digital art work "funeral of my goldfish"

From the deepest point of art, there is no essential difference between digital art and traditional art. 
Instead, they are only the different tools of creation. There are many traditional art works that only express the author's inner feelings, for example, Chinese traditional literati painting. There are also many such works of digital art, especially by those artists who have been educated in traditional painting and master digital art skills. They created a lot of pure works of art expressing their personal feelings through computers, without any commercial purpose.

Figure 1 shows a typical digital art work. This work is not a mere imitation of a natural image, but has a rich painting language and profound meaning, and it expresses a philosophical thinking in an artistic way.

From another perspective, since the digital art works can be infinitely copied, it is not easy for authors to obtain economic benefits from the copyright of original works, therefore, it seems that the creative motives of digital art are more pure and thorough than traditional painting, and it is closer to the essence of artistic creation

However, judging from the status quo of practice, at present, digital art is undoubtedly more used in advertising, packaging and other practical art fields, but it still cannot obliterate or diminish the artistry of the visual effects of the work. In the era without computers, traditional art has also been applied to these practical art fields. In commodity economy society, art works that do not have pure artistic criticism are also considered as one of the works of art. The key to the artistry of art works lies in whether they can bring spiritual enjoyment to people. No matter traditional art or digital art, when they can't meet people's need for any material, if it can still bring aesthetic pleasure to people and satisfy the spiritual beauty of people, then these works can be said to have a certain artistic spirit.

The reason why digital art can be so widely spread in such a short time is that their brand new artistic form coincides with the aesthetic psychology of contemporary people. Therefore, as same as traditional art works, digital works can also convey human emotions and inspire wisdom through works.

The concrete manifestation of digital art in artistic spirit is mainly in the following aspects:

\subsection{It embodies the western art concept of seeking truth.}

Digital art is a high unity of contemporary high technology and art. The humanistic spirit of western culture emphasizes the conquest and exploration of human nature, while oriental culture emphasizes the unity of heaven and human, and pursues emotional intuition and understanding. So in artistic creation, the western is more in pursuit of the unity of beauty and truth, which embodies western scientific and rational thinking. In terms of painting art, the west has long been keen on the imitation of nature, and the works are partial to the reappearance of the realistic object. With the application of computer technology to art creation, the powerful and accurate computing power of the computer has pushed the traditional idea of "seeking truth" to a new peak.

A still life painting by computer is shown as Figure 2.

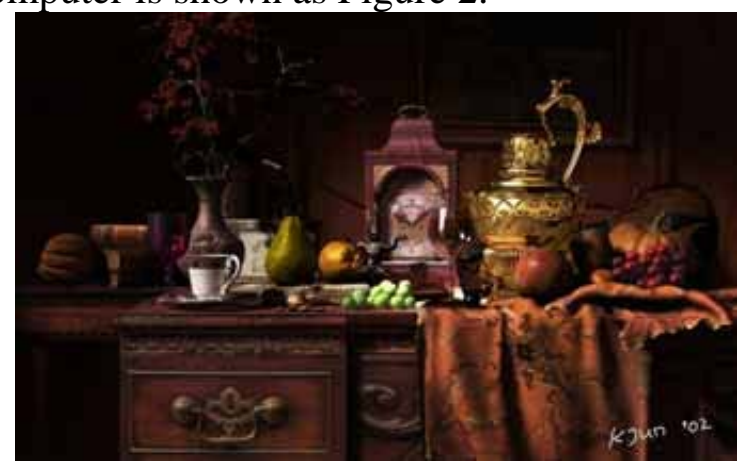

Figure 2. Digital art work "Still”

This work has a strong style of classical oil painting, and meanwhile, realistic color and accurate modeling reveal the magic charm of digital art.

Of course, too much pursuit of reality in digital art will also cause the appreciation fatigue of appreciers, leading to the restriction of readers' imagination of space, as well as aesthetic pleasure brought about by imagination, and even readers may get into a passive aesthetic automation mire. So 
it needs to be guarded and avoided.

\subsection{It promotes the popularization of artistic creation.}

The artistic spirit of digital art also manifests in the popularization of artistic creation. Art creation is not some patented product of genius, but anyone has the potential of artistic creation, although the transformation from potential to talent is restricted by certain conditions. With the help of software tools, digital art makes the training of long-term manual craftsmanship necessary for an artist in the past no longer an obstacle to art creation. For example, Traditional geometry sketch training requires a lot of practice to master, but it comes to a simple command by using certain software, so that it help save the time and effort that the creators spend on mastering basic skills. Creators are allowed to have the freedom to express their artistic ideas freely, and even create unexpected dream works.

Meanwhile, with the popularity of computers, digital art thoroughly brings art into the world of creative subjects. It inspires many people with artistic expression desires to create passion for digital art, and art creation is no longer the patent of a few artists. To some extent, art creation goes into mass life. For ordinary people, from appreciation to participation, from feeling beauty to creating beauty, that is not only a qualitative change in aesthetic cultural life, but also the liberation of one's own spirit.

In digital art, the boundaries between traditional professional painters and amateur painters began to disappear. Not only does art creation become more freedom, but also the dissemination and communication of works is also more convenient. People's creative complex and publishing desire have been released unprecedentedly, so there are a large number of digital art works circulating on the Internet that do not know the creator's name.

Certainly, the popularization of digital art creation and the networking of communication channels of works is like web writing, which will inevitably lead to the uneven appearance of various works displayed in front of people, and there are even some vulgar works circulating on the Internet, so this is what we need to know critically.

\subsection{It strengthens the spirit of humanistic care.}

Digital arts, like traditional arts, belong to the category of humanities, and its core meaning lies in the embodiment of its humanistic spirit. With the coming of the age of reading, the role of digital art in the dissemination of humanistic spirit is more and more important. Humanistic spirit is a universal human self-care, showing the maintenance, pursuit and concern for human dignity, value and fate. It is highly valued for all kinds of spiritual and cultural phenomena left by human beings, as well as the affirmation and molding of an all-round development of ideal personality. Humanities is a knowledge education system that focuses on the expression of humanistic spirit, which focuses on human values and mental performance. In a sense, the reason why people are the spirit of all things, is that they have their own unique spiritual culture, and such humanistic spirit is precisely the internal spirit of digital art.

Digital art is not a solution to the traditional art at the spiritual level, instead, under the condition of traditional art becoming more and more depressed, digital art brings a new breakthrough and innovation to the field of visual art. To be accurate, it is technology progress and market motivation that brings beautiful prospects to the current art creation. It satisfies people's profound needs in the spiritual world, and further strengthens human thinking on their own destiny. More importantly, it most accurately reflects the changes of our time.

Digital arts as the crystallization of artistic creative thinking and high-tech means, not only greatly enriches the world of contemporary visual arts, but also helps to communicate human spirit and emotion. The digital art shows a large number of unimaginable virtual forms that surpass the real space through the means of computer technology, to make people's aesthetic tastes in the spiritual field slowly change, and expand and extend people's diverse spiritual needs. Many contemporary experimental artists and designers are using computer and internet media technologies to create a large number of new art forms in the areas of music, sculpture, architecture, graphic design, film and television, etc. that pay attention to contemporary society and reflect on the future of mankind. A variety of fine art works play an increasingly important role in people's spiritual life, and affects the 
values and aesthetic orientation of contemporary people.

For instance, there is a disaster film in the U.S. that reflects the climate change caused by global warming, called "The Day After Tomorrow". In the movie, digital art creation methods are widely used to simulate realistic scenes of disastrous weather, including hailstones, tsunamis, tornadoes, etc. The verisimilitude scene makes the viewer stare, shows as Figure 3.

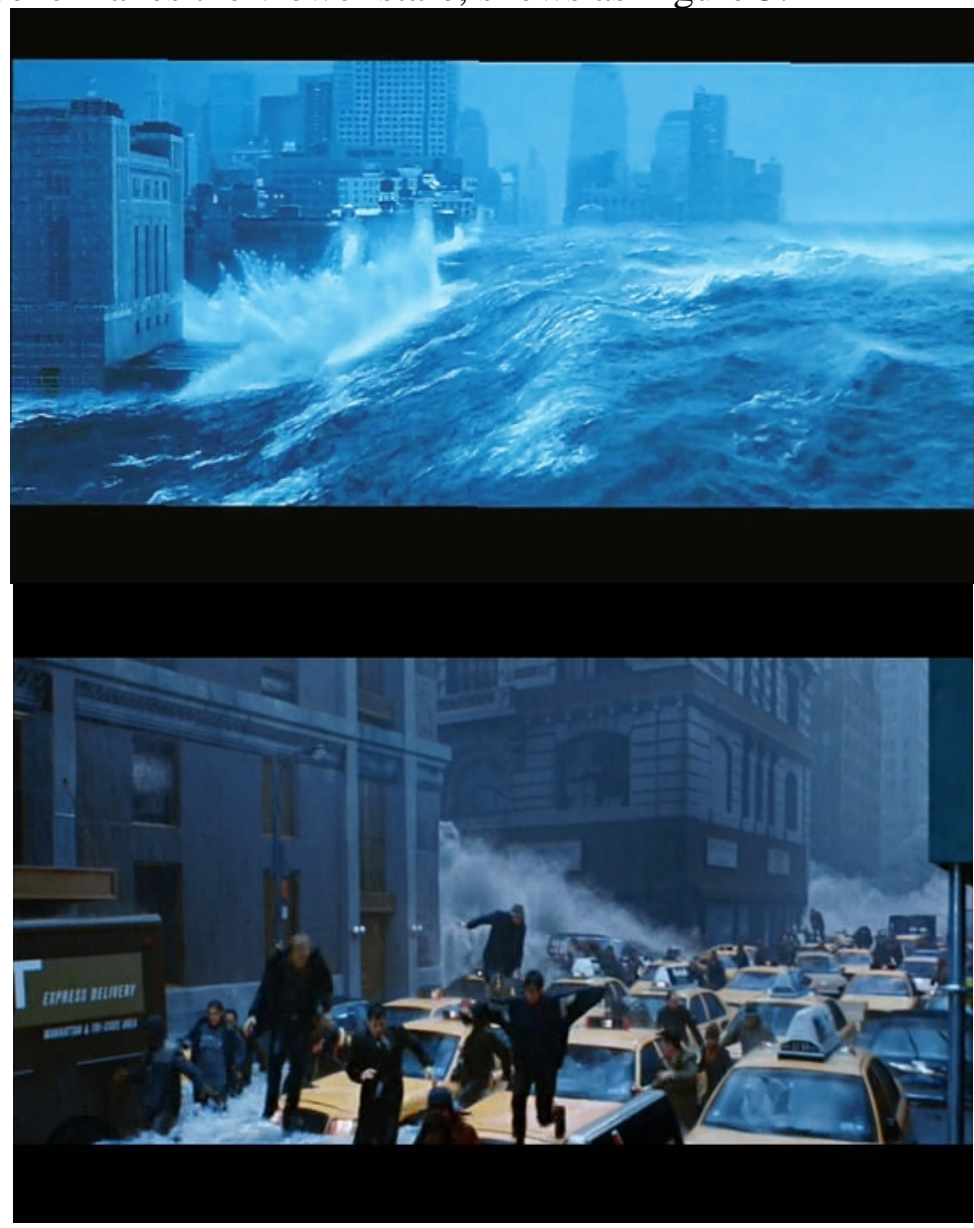

Figure 3. Stills of "The Day after Tomorrow"

Imagine that without the help of digital art, only by text description, readers' imagine or display by traditional paintings, can the picture bring people such a shocking artistic appeal? Can it really touch human's deep thinking about their future destiny? Obviously not! It is digital art that deepens our understanding of the unknown world, and strengthens the educational function of art. Moreover, computer technology is the foundation of future social structure and living space form, and it also plays an important role in the construction of the spiritual level of the future society. It will eventually enhance human spirit and artistic activities to a higher level in history and evolve into a more colorful form.

\section{Reflection on Digital Art Creation}

With the popularization of digital technology in art creation, there are some problems that deserve our careful reflection.

Firstly, some people do not correctly treat the relationship between people and computers in digital art creation, and the think that with the help of computer, they just need to combine all kinds of original materials to conduct art creation, and there is no need for good ideas. Actually, computer can only help artists to complete the artistic conception, and replace people to do some repetitive work, but it can never replace the artist's creative thinking, because the computer itself has neither emotion nor active thinking ability. However, the artist's artistic cultivation and artistic thought are the most fundamental. If the work does not contain the profound humanistic spirit and artistic thought that the 
artist wants to express, then digital art can only become a kind of "bizarre trick".

Secondly, if you are overly dependent on technology, creators can easily lose the spiritual value and autonomy of life in artistic creation. Software developers continually release new software versions for commercial benefit, so it can easily lead people to study all kinds of software skills throughout the day, but ignore the research and grasp of the law of artistic creation itself. We must know that, technology is only a necessary guarantee for the realization of the creative idea, but it's never the most important. Relying too much on digital technology will only constrain human imagination, and the trailblazers and slaves that eventually make people machines.

Thirdly, we can't contrast digital art with traditional art. Digital art is the inheritance and development of traditional art. The digital arts only implement a revolution in traditional arts in terms of specific operating techniques, while in terms of color, composition and other creative principles, digital art undoubtedly inherits the clothes of traditional art, so only by mastering the creation theory of traditional art can we better create digital art. Conversely, we also can't exclude digital art, because whether you like it or not, it has been an indisputable fact that digital art is the main realization tool in the field of art design.

\section{Conclusion}

Overall speaking, the digitalization of art is a double-edged sword. It has opened an unprecedented space for the production and dissemination of literature and art, but it also lays a variety of cultural traps at the same time. Art creation, especially in the field of art and design, is becoming more and more dependent on computer technology, and this may be an irreversible trend of the times. However, for digital art, we should keep a clear head. While making use of all kinds of conveniences and benefits brought by science and technology, we should always maintain the vigilance of humanism and the critical spirit of rational culture, and maintain the embodiment of humanity in artistic creation.

\section{References}

[1] Marcos A F, Branco P S, Zagalo N T. The Creation Process in Digital Art[M]// Handbook of Multimedia for Digital Entertainment and Arts. 2009:601-615.

[2] Zhang G. Difficult Condition of Digital Copy and of Digital Art Creation[J]. 2009.

[3] Zhang X. Elements in the Creation of Digital Media Art[J]. Journal of Tianjin Academy of Fine Arts, 2010.

[4] Maallem H B, Richard P, Ferrier J L, et al. Can Virtual or Digital Creation be Considered as Art or Technique ?[J]. 2013.

[5] RESEARCH ON DIGITAL MEDIA ART CREATION BASED ON VIRTUAL REALITY TECHNOLOGY[J]. Design, 2017.

[6] Digital Art and Creation Ideas[J]. Chinese Art, 2012. 